\title{
BMJ Open Independent associations of blood pressure and body mass index with interatrial block: a cross-sectional study in general Chinese population
}

Guozhe Sun, Ying Zhou, Ning Ye, Shaojun Wu, Yingxian Sun

To cite: Sun G, Zhou Y, Ye N, et al. Independent associations of blood pressure and body mass index with interatrial block: a crosssectional study in general Chinese population. BMJ Open 2019;9:e029463. doi:10.1136/ bmjopen-2019-029463

- Prepublication history for this paper is available online. To view these files please visit the journal online (http://dx.doi org/10.1136/bmjopen-2019029463).

Received 28 January 2019

Revised 31 May 2019

Accepted 7 June 2019
Check for updates

(C) Author(s) (or their employer(s)) 2019. Re-use permitted under CC BY-NC. No commercial re-use. See rights and permissions. Published by BMJ.

Department of Cardiovascular Medicine, First Hospital of China Medical University, Shenyang, Liaoning, China

Correspondence to Professor Yingxian Sun; cmu1h_syx@126.com

\section{ABSTRACT}

Objectives This current study was performed to characterise the independent associations of obesity and hypertension with interatrial block (IAB) after adjusting for cardiovascular risk factors, echocardiographic left atrial diameter (LAD) and left ventricular mass index (LVMI) in a large general Chinese population.

Design A cross-sectional study.

Setting and participants A total of 11956 permanent residents ( $\geq 35$ years of age) from Liaoning Province in China were included in this study. Following the completion of a questionnaire, the enrolled participants were subjected to physical examinations, laboratory analyses, ECG and echocardiogram. Linear and logistic regression analyses were performed to evaluate the associations of hypertension and obesity with IAB.

Outcome measures $I A B$ was defined as a prolongation of the $P$ wave duration $\geq 120$ ms on a digital 12-lead ECG. Results The prevalence of IAB in hypertensive individuals was higher than the normotensive in both men (9.5 vs $5.9 \% ; p<0.001$ ) and women (6.6 vs $3.6 \% ; p<0.001)$. In addition, the prevalence of $I A B$ exhibited a sharp increase with advancing body mass index (BMI) in both men (from $4.9 \%$ to $13.0 \%$ ) and women (from $3.5 \%$ to $6.9 \%$ ) (psfor trend $<0.001)$. Multiple relevant clinical covariates, echocardiographic LAD and LVMI were adjusted in the multivariate linear and logistic regression analyses. The results revealed that systolic blood pressure, diastolic blood pressure and BMI were all independently associated with $P$ wave duration $(\beta=0.02,0.09$ and 0.25 , respectively all ps $<0.005)$. Furthermore, hypertension was found to be independently associated with $I A B(O R=1.27 ; p=0.018)$, while both overweight and obesity exhibited higher odds of IAB (OR=1.42 and 1.67, respectively; $p s<0.005)$, compared with $\mathrm{BMl}<24.0 \mathrm{~kg} / \mathrm{m}^{2}$.

Conclusions The key findings of this study highlighted that hypertension and overweight/obesity were independently and significantly associated with IAB in general Chinese population.

\section{INTRODUCTION}

Interatrial block (IAB) is characterised by the presence of a prolonged $\mathrm{P}$ wave exceeding $120 \mathrm{~ms}$ on a 12-lead ECG. ${ }^{1}$ Accumulating reports have indicated that the prevalence of $\mathrm{IAB}$ is frequently underappreciated in clinical

\section{Strengths and limitations of this study}

- The current study evaluated the independent associations of hypertension and overweight/obesity with interatrial block.

- This was a large population-based study, providing adequate data and sample size to delineate the study objective.

- Digital ECG was an important strength since automatic measures had superior validity and reliability compared with manual readings.

- Besides multiple clinical covariates, echocardiographic left atrial diameter and left ventricular mass index were also adjusted in multivariate logistic regression analyses.

- This was a cross-sectional study and further prospective ones should be conducted.

practice, particularly in the male and ageing populations. ${ }^{2-4}$ Further worsening the magnitude, IAB has been linked with numerous medical conditions including atrial arrhythmias, ${ }^{5-7}$ abnormal left atrial function ${ }^{8}$ and thromboembolic ischaemic stroke. ${ }^{9-12} \mathrm{~A}$ follow-up study demonstrated that advancing $\mathrm{P}$ wave durations are significantly associated with increasing cardiovascular and all-cause mortality. ${ }^{13}$ Therefore, as a potentially crucial predictor of long-term patient outcome, additional efforts are necessitated in order to further elucidate the prevalence of IAB and its associated risk factors.

Obesity and hypertension are two highly prevalent conditions which remain to be burden on clinical resources, and remarkably, have been reported to possibly lead to left atrial enlargement. Previous studies have highlighted that both obesity and hypertension serve as risk factors for IAB, but the majority of these studies were performed in general hospitals with patients admitted for non-acute issues with limited sample sizes. ${ }^{1415}$ More notably, the study conducted by Atherosclerosis Risk in Communities (ARIC) 
demonstrated that both obesity and metabolic syndrome (especially with hypertension) are correlated with IAB, independent of age and other cardiovascular risk factors. ${ }^{16}$ However, these studies failed to take left atrial size into account. Hence, the current study sought to examine whether these associations with IAB were dependent on echocardiographic left atrial diameter (LAD) changes, a novel investigation that is yet to be conducted. In addition, no previous studies have incorporated large sample size studies emphasising the risk factors contributing to IAB in Chinese population. Thereby, the current study aimed to assess the independent associations of obesity and hypertension with IAB after adjusting for cardiovascular risk factors and echocardiographic changes in general Chinese population using a large-scale cross-sectional study.

\section{MATERIALS AND METHODS \\ Study population}

Between January 2013 and August 2013, a representative sample of men and women from Liaoning province of China were evaluated for cardiovascular risk factors (primarily hypertension) using a multi-stage, random, stratified, cluster-sampling scheme, referred to as the Northeast China Rural Cardiovascular Health Study. The current study intentionally enrolled a representative sample aged $\geq 35$ years, due to its purpose of evaluating hypertension and related cardiovascular risk factors. Within the Liaoning province, three counties (Dawa, Zhangwu and Liaoyang) were selected from the eastern, southern and northern regions, where the greater majority of residents are agricultural labourers. One township near a city in each county was randomly selected, totaling 3 townships and 5-8 villages from each township were randomly selected, with a total of 18 rural villages finally selected. Those who were pregnant, suffering from cancers or mental disorders were excluded from the current study.

All eligible permanent residents $\geq 35$ years of age from each village $(\mathrm{n}=14016)$ were initially recruited, and a total of $11956(85.3 \%)$ participants completed the study. Subjects with incomplete data, poor ECG quality, atrial fibrillation (AF)/flutter, paced rhythm, Wolff-Parkinson-White syndrome or congenital heart diseases were excluded from the study, leaving a total of 11264 participants for the final analyses. All participants signed written consent after they had been informed of the study's objectives, benefits, medical procedures and confidentiality safeguards for personal information. Also, informed consent was obtained from the proxies of participants who were illiterate.

\section{Data collection and measurements}

Data were collected during a single clinic visit by cardiologists and trained nurses using a standard questionnaire with face-to-face interviews. All the potential investigators had received training in relation to the objectives of the study, how to perform the questionnaire, the standard methods of measurement, the importance of standardisation, as well as the finer details of the study procedures. Only those who earned a perfect score on a post-training test were permitted to participate as study investigators. During the process of data collection, the investigators were provided with additional instructions and support. Data on demographic characteristics, medical history and lifestyle risk factors were obtained using the abovementioned interviews with a standardised questionnaire. A central steering committee with a subcommittee for quality control ensured that all data were collected in accordance with the aforementioned standards.

According to the guidelines of the American Heart Association, blood pressure (BP) was measured three times at 2 min intervals, with a resting period of at least 5 min using a standardised automatic electronic sphygmomanometer (HEM-907; Omron, Kyoto, Japan). Independently, two doctors checked the calibration of the Omron device every month using a standard mercury sphygmomanometer in accordance with the British Hypertension Society protocol. ${ }^{17}$ All participants were directed to avoid caffeinated beverages and exercise at least 30 min prior to evaluation. During BP measurement, the participants were seated with their arms supported at the level of their hearts. The mean value of three BP measurements were calculated and used in all the subsequent analyses. Hypertension was defined by the criteria widely employed and considered to be the worldwide standard in epidemiological research studies: a systolic blood pressure (SBP) $\geq 140 \mathrm{~mm} \mathrm{Hg}$ and/or diastolic blood pressure (DBP) $\geq 90 \mathrm{~mm} \mathrm{Hg}$ and/or the use of antihypertensive medications. All participants were classified into the following groups based on mean SBP/DBP values and the most recent 2017 American College of Cardiology/ American Heart Association guidelines ${ }^{18}$ : (a) normal: SBP $<120 \mathrm{~mm} \mathrm{Hg}$ and DBP $<80 \mathrm{~mm} \mathrm{Hg}$, (b) elevated BP: SBP $120-129 \mathrm{~mm} \mathrm{Hg}$ and DBP $<80 \mathrm{~mm} \mathrm{Hg}$, (c) stage 1 hypertension: SBP $130-139 \mathrm{~mm} \mathrm{Hg}$ or DBP $80-89 \mathrm{~mm} \mathrm{Hg}$ and (d) stage 2 hypertension: SBP $\geq 140 \mathrm{~mm} \mathrm{Hg}$ or DBP $\geq 90 \mathrm{~mm} \mathrm{Hg}$. During the course of the study, subjects who were taking anti-hypertensive medication and had a history of hypertension were considered to be at stage 2 hypertension as their BP levels would have exceeded 140/90 mm Hg during their initial hypertension diagnosis in accordance with the previous criteria. Weight and height were measured to the nearest $0.1 \mathrm{~kg}$ and $0.1 \mathrm{~cm}$, respectively, with all participants given lightweight clothing and evaluated barefoot. The body mass index (BMI) was calculated as weight in kilograms divided by the square of height in metres $\left(\mathrm{kg} / \mathrm{m}^{2}\right)$. As recommended by the Working Group on Obesity in China, overweight was defined as a BMI of $24.0-27.9 \mathrm{~kg} / \mathrm{m}^{2}$ and obesity as a BMI of $28.0 \mathrm{~kg} / \mathrm{m}^{2}$ or higher. ${ }^{19}$

Fasting blood samples were collected in the morning after $\geq 8$ hour of fasting. Blood samples were collected from the antecubital vein using BD Vacutainer tubes containing EDTA (Becton, Dickinson and Co., Franklin 


\begin{tabular}{|c|c|c|c|}
\hline Variable & $\begin{array}{l}\text { IAB - } \\
(n=10553)\end{array}$ & $\begin{array}{l}\mid A B+ \\
(n=711)\end{array}$ & $P$ value \\
\hline Age, years & $53.6 \pm 10.6$ & $55.5 \pm 10.2$ & $<0.001$ \\
\hline Male & $4724(44.8)$ & $402(56.5)$ & $<0.001$ \\
\hline Race of Han & 10009 (94.8) & $668(94.0)$ & 0.300 \\
\hline Height, $\mathrm{cm}$ & $160.4 \pm 8.2$ & $163.1 \pm 8.1$ & $<0.001$ \\
\hline Weight, kg & $63.7 \pm 11.3$ & $69.2 \pm 11.6$ & $<0.001$ \\
\hline BMI, kg/m² & $24.7 \pm 3.7$ & $25.9 \pm 3.6$ & $<0.001$ \\
\hline BMI category, $\mathrm{kg} / \mathrm{m}^{2}$ & & & $<0.001$ \\
\hline$<24$ & $4752(45.0)$ & $206(29.0)$ & \\
\hline $24-28$ & 3989 (37.8) & $315(44.3)$ & \\
\hline$\geq 28$ & 1812 (17.2) & $190(26.7)$ & \\
\hline SBP, $\mathrm{mm} \mathrm{Hg}$ & $141.3 \pm 23.3$ & $149.4 \pm 24.9$ & $<0.001$ \\
\hline DBP, mm Hg & $81.7 \pm 11.6$ & $85.7 \pm 12.7$ & $<0.001$ \\
\hline Hypertension & $5288(50.1)$ & $459(64.6)$ & $<0.001$ \\
\hline BP category & & & $<0.001$ \\
\hline Normal & 1657 (15.7) & $67(9.4)$ & \\
\hline Elevated & $1333(12.6)$ & $54(7.6)$ & \\
\hline $\begin{array}{l}\text { Stage } 1 \\
\text { Hypertension }\end{array}$ & $2275(21.6)$ & $131(18.4)$ & \\
\hline $\begin{array}{l}\text { Stage } 2 \\
\text { Hypertension }\end{array}$ & $5288(50.1)$ & $459(64.6)$ & \\
\hline $\mathrm{FBG}, \mathrm{mmol} / \mathrm{L}$ & $5.88 \pm 1.61$ & $6.08 \pm 1.75$ & 0.003 \\
\hline Diabetes & 1067 (10.1) & 89 (12.5) & 0.041 \\
\hline $\mathrm{TC}, \mathrm{mmol} / \mathrm{L}$ & $5.22 \pm 1.07$ & $5.37 \pm 1.31$ & 0.003 \\
\hline $\mathrm{TG}, \mathrm{mmol} / \mathrm{L}$ & $1.62 \pm 1.49$ & $1.86 \pm 1.65$ & $<0.001$ \\
\hline LDL-C, mmol/L & $2.92 \pm 0.81$ & $3.03 \pm 0.93$ & 0.001 \\
\hline HDL-C, mmol/L & $1.41 \pm 0.38$ & $1.37 \pm 0.38$ & 0.002 \\
\hline SUA, mg/dL & $4.87 \pm 1.42$ & $5.22 \pm 1.45$ & $<0.001$ \\
\hline Current smoker & 3712 (35.2) & $245(34.5)$ & 0.699 \\
\hline Current drinker & 2313 (21.9) & $196(27.6)$ & $<0.001$ \\
\hline Education level & & & 0.004 \\
\hline$\leq$ Primary school & $5274(50.0)$ & $348(48.9)$ & \\
\hline Middle school & $4328(41.0)$ & $273(38.4)$ & \\
\hline 2High school & $951(9.0)$ & $90(12.7)$ & \\
\hline Family income, CNY/y & & & 0.281 \\
\hline$\leq 5000$ & $1317(12.5)$ & 85 (12.0) & \\
\hline $5000-20000$ & $5787(54.8)$ & $373(52.5)$ & \\
\hline$>20000$ & 3449 (32.7) & $253(35.6)$ & \\
\hline LAD, mm & $33.6 \pm 3.9$ & $35.3 \pm 4.6$ & $<0.001$ \\
\hline LAE & $968(9.4)$ & $141(20.4)$ & $<0.001$ \\
\hline LVMI, g/m² & $81.7 \pm 19.0$ & $87.7 \pm 21.5$ & $<0.001$ \\
\hline LVH & 1047 (10.3) & $111(16.3)$ & $<0.001$ \\
\hline $\mathrm{P}$ wave duration, ms & $99.4 \pm 12.2$ & $125.6 \pm 7.7$ & $<0.001$ \\
\hline Heart rate, bpm & $71.5 \pm 12.0$ & $73.2 \pm 15.5$ & 0.004 \\
\hline $\begin{array}{l}\text { Anti-arrhythmic } \\
\text { medication }\end{array}$ & $58(0.5)$ & $9(1.3)$ & 0.037 \\
\hline
\end{tabular}

Continued

\begin{tabular}{lccc}
\hline Table 1 Continued & \multicolumn{1}{l}{} \\
\hline Variable & $\begin{array}{l}\text { IAB }- \\
(\mathbf{n}=\mathbf{1 0 5 5 3 )}\end{array}$ & $\begin{array}{l}\text { IAB + } \\
(\mathbf{n}=\mathbf{7 1 1})\end{array}$ & P value \\
\hline $\begin{array}{l}\text { Anti-hypertensive } \\
\text { medication }\end{array}$ & $1641(15.6)$ & $174(24.5)$ & $<0.001$ \\
$\begin{array}{l}\text { History of MI } \\
\text { History of HF }\end{array}$ & $117(1.1)$ & $13(1.8)$ & 0.082 \\
$\begin{array}{l}\text { Mitral stenosis/ } \\
\text { regurgitation }\end{array}$ & $158(0.8)$ & $10(1.4)$ & 0.083 \\
\hline
\end{tabular}

Data are expressed as mean \pm SD or $\mathrm{n}(\%)$.

$\mathrm{BMI}$, body mass index; BP, blood pressure; CNY, China Yuan; DBP, diastolic blood pressure; FBG, fasting blood glucose; $\mathrm{HDL}-\mathrm{C}$, high-density lipid cholesterol; HF, heart failure; IAB, interatrial block; LAD, left atrial diameter; LAE, left atrial enlargement; LDL-C, low-density lipid cholesterol; LVH, left ventricular hypertrophy; LVMI, left ventricular mass index; MI, myocardial infarction; SBP, systolic blood pressure; SUA, serum uric acid; TC, total cholesterol; TG, triglycerides.

Lakes, NJ, USA). Subsequently, serum was isolated from whole blood, with all serum samples subsequently frozen at $-20^{\circ} \mathrm{C}$ for testing at a central, certified laboratory. Fasting blood glucose (FBG), total cholesterol (TC), triglycerides (TG), high-density lipid cholesterol (HDL-C), low-density lipid cholesterol (LDL-C), serum uric acid (SUA) and other routine blood biochemical indices were analysed enzymatically using an auto-analyzer (Olympus AU640 Auto-Analyzer; Olympus Corp., Kobe, Japan). According to the criteria issued by WHO, diabetes mellitus was defined as a FBG $\geq 7.0 \mathrm{mmol} / \mathrm{L}$ and/ or patients currently being treated for diabetes. ${ }^{20}$

In addition, 12-lead resting, 10-s ECGs were performed on all participants by well-trained cardiologists using an electrocardiography machine (MAC 5500; GE Healthcare, Little Chalfont, Buckinghamshire, UK). The results were automatically analysed by the MUSE Cardiology Information System (V.7.0.0; GE Healthcare). The P wave in each lead was defined as the initial upward point or downward deflection from the isoelectric line to the point of the initial baseline. For the calculation of the $\mathrm{P}$ wave duration, onsets were defined as the earliest deflection in any lead and offsets were defined as the latest deflection in any lead. Employing the most recent consensus guidelines, ${ }^{1}$ IAB was defined as a prolonged $\mathrm{P}$ wave duration $\geq 120 \mathrm{~ms}$ on a 12-lead ECG in the current study.

Echocardiograms were obtained using a commercially available Doppler echocardiograph (Vivid; GE Healthcare) with a 3.0-MHz transducer. Echocardiogram analyses and readings were performed by three separate doctors, all of whom were specialised in echocardiography, while two other specialists were called in case of any questions or uncertainties. LAD in the current study was defined as the left atrial anteroposterior measurement in the parasternal long-axis view according to the recommendations of the American Society of Echocardiography. ${ }^{21}$ The reported LAD values in our study were not indexed by body surface area. Left ventricular mass 

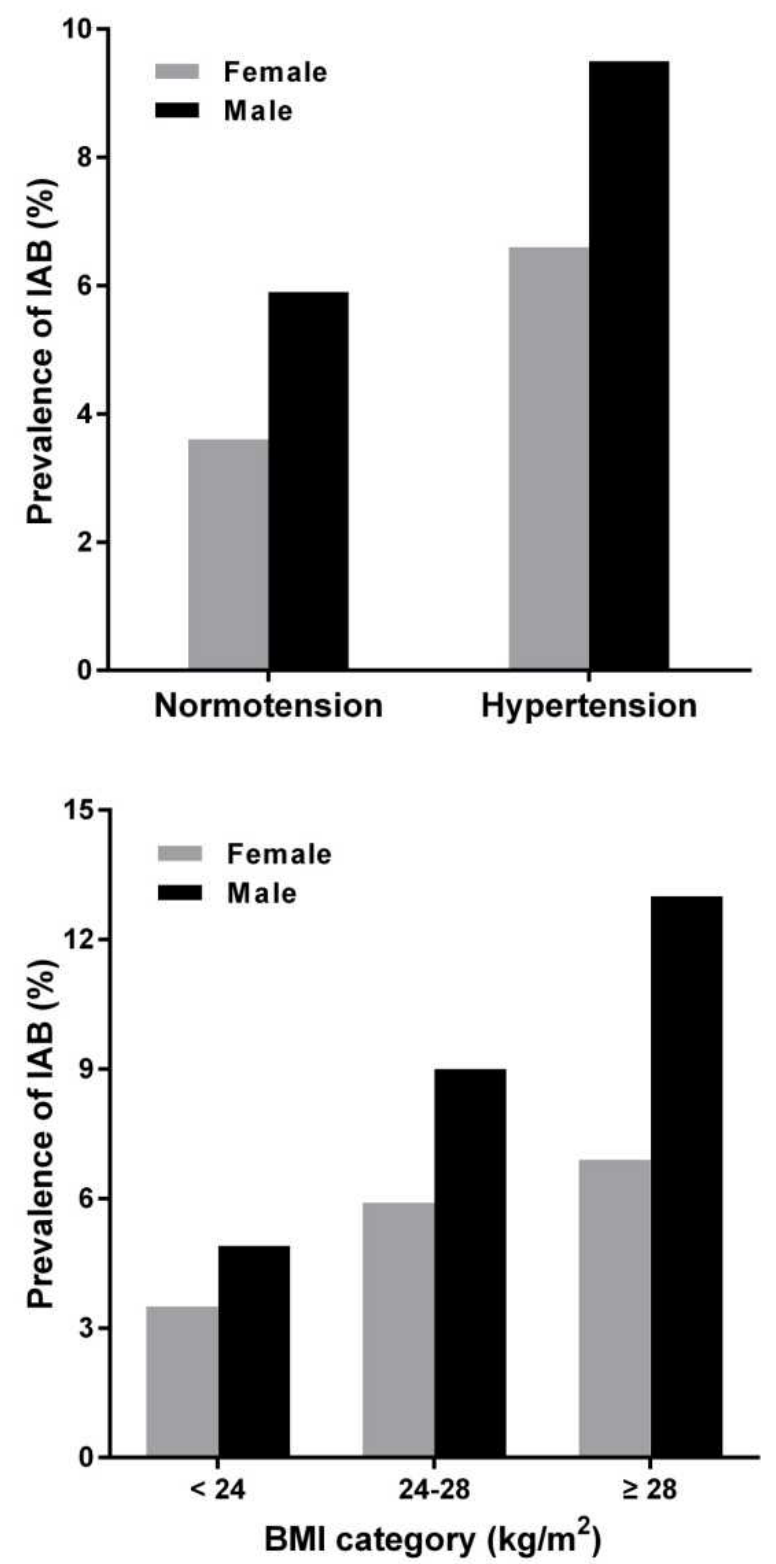

Figure 1 Gender-specific prevalence of interatrial block (IAB) by hypertension and body mass index (BMI) category.

index (LVMI) was calculated based on body surface area, while left ventricular hypertrophy $(\mathrm{LVH})$ was defined as a LVMI $>115 \mathrm{~g} / \mathrm{m}^{2}$ in men and $>95 \mathrm{~g} / \mathrm{m}^{2}$ in women.

\section{Statistical analysis}

All statistical analyses were performed using SPSS V.17.0 statistical software (SPSS, Inc, Chicago, IL, USA). Differences between groups were compared using a two-tailed Student's t-test for continuous variables and a $\chi^{2}$ test for categorical variables. IAB prevalence by BMI category and hypertension were calculated and presented accordingly. Multivariate linear regression analyses were performed to identify the linear correlation among BP, BMI and $\mathrm{P}$ wave duration. Multivariate logistic regression analyses were conducted to evaluate the link between hypertension and obesity with IAB. Data were expressed as OR and 95\% CI,

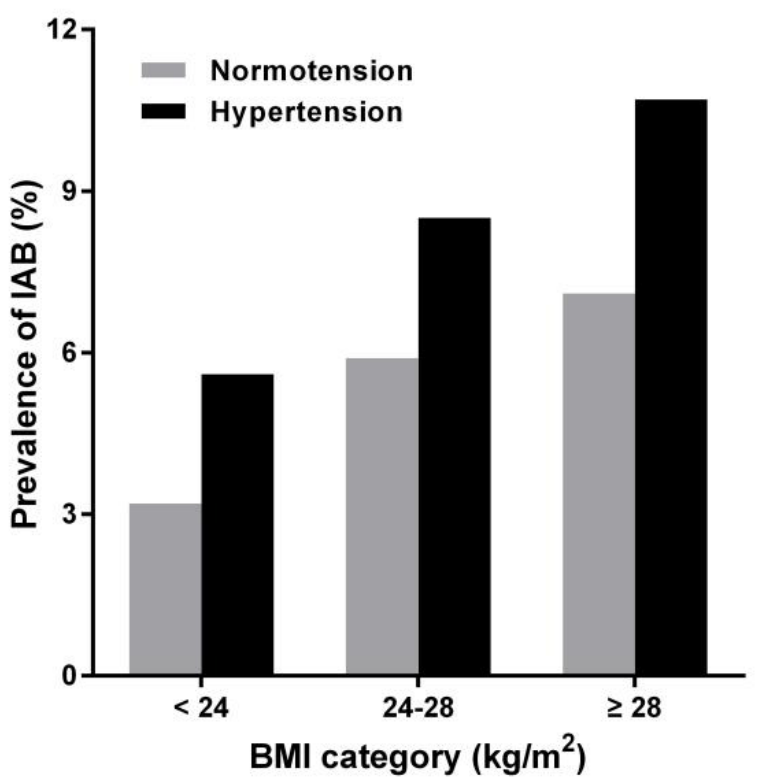

Figure 2 Prevalence of interatrial block (IAB) for body mass index (BMI) category by hypertension.

$\beta$, mean $\pm \mathrm{SD}$ or frequency and percentages; $\mathrm{A} p$ value $<0.05$ was considered to be statistically significant.

\section{Patient and public involvement}

No patients were involved in setting the research questions or the outcome measures, nor were they involved in the design or performance of the study. No participants or patients were asked to advise on the interpretation or writing up of results. No plans were set in place to disseminate the results of the research to study participants.

\section{RESULTS}

\section{Characteristics of the study population}

A total of 11264 participants were included for final analyses, comprising of 5126 men and 6138 women with a mean age of 53.8 years. The general prevalence of IAB was calculated to be $6.3 \%(711 / 11264)$ within the total population, which was significantly higher in subjects with left atrial enlargement (LAE) than those without (12.7 vs $5.6 \% ; \mathrm{p}<0.001)$. The subjects with $\operatorname{IAB}(\mathrm{n}=711)$ were significantly older and exhibited higher BMI, SBP, DBP, FBG, TC, TG, LDL-C, SUA, LAD, LVMI and heart rate values compared with those with normal $\mathrm{P}$ wave durations (all ps<0.005) (table 1). Furthermore, the participants with IAB had significantly higher percentages of men, current drinker, anti-arrhythmic medication and anti-hypertensive medication, and higher prevalences of obesity, hypertension, diabetes mellitus, LAE and LVH (all ps $<0.05$ ). Notably, HDL-C levels were found to be lower in subjects with IAB than those without IAB $(\mathrm{p}=0.002)$. No significant differences were detected regarding smoking status between the two groups $(p=0.699)$. Furthermore, IAB participants were identified with a relatively higher prevalence of myocardial infarction (MI), heart failure 
Table 2 Multivariate linear regression analyses for associations between BP, BMI and P wave duration

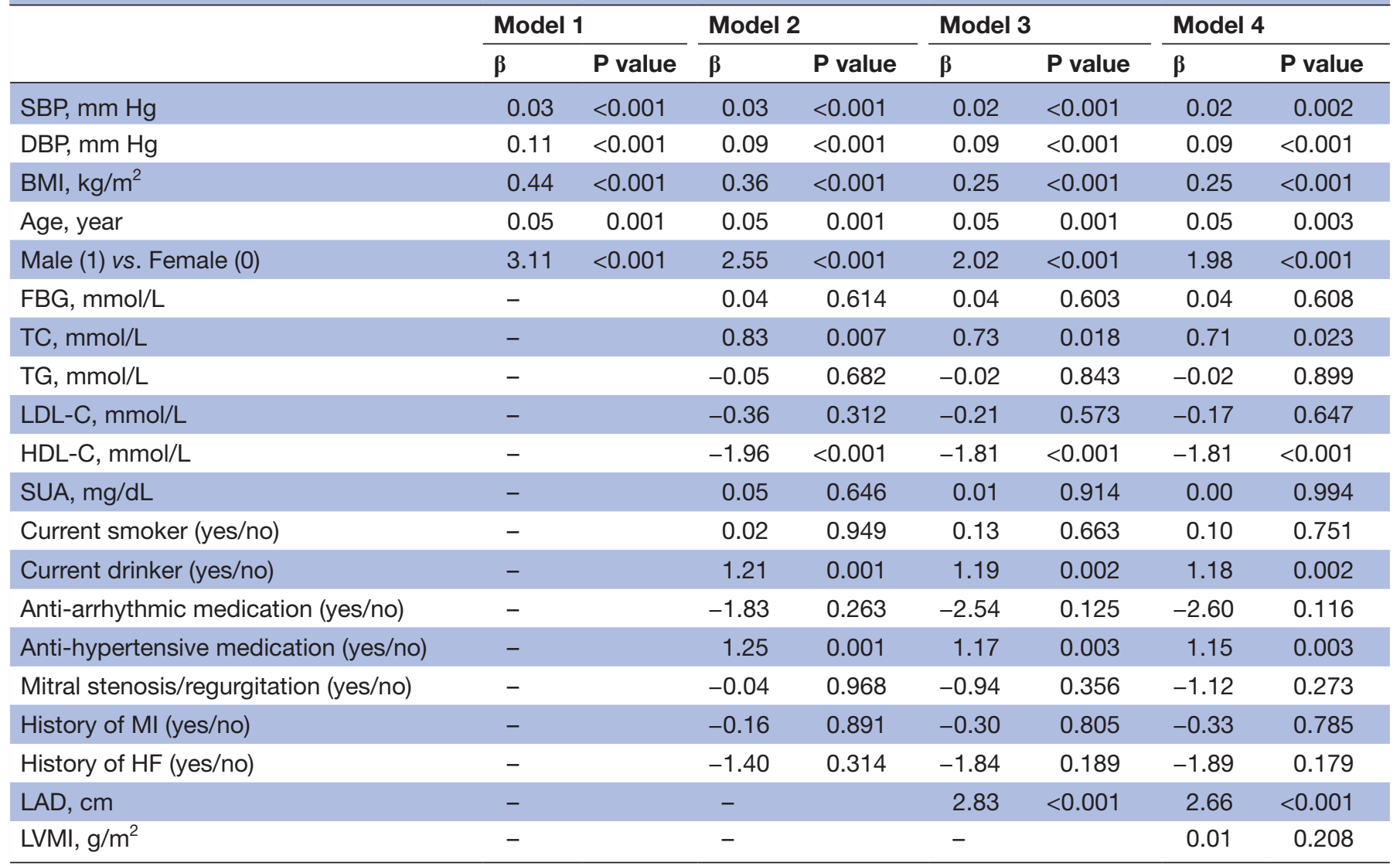

Model 2: additional variables including FBG, plasma lipids, SUA, smoking, drinking, education, income, anti-arrhythmic medication, antihypertensive medication, mitral stenosis/regurgitation, and history of MI and HF; model 3: additional variable LAD; model 4: additional variable LVMI.; Note, model 1: SBP, DBP, BMI, age, gender and race in the multivariate regression.

BMI, body mass index; BP, blood pressure; CNY, China Yuan; DBP, diastolic blood pressure; FBG, fasting blood glucose; HDL-C, highdensity lipid cholesterol; HF, heart failure; IAB, interatrial block; LAD, left atrial diameter; LAE, left atrial enlargement; LDL-C, low-density lipid cholesterol; LVH, left ventricular hypertrophy; LVMI, left ventricular mass index; MI, myocardial infarction; SBP, systolic blood pressure; SUA, serum uric acid; TC, total cholesterol; TG, triglycerides.

(HF) and mitral stenosis/regurgitation, even though no statistical significance was detected (all ps>0.05).

\section{IAB prevalence by hypertension and BMI category}

The gender-specific prevalence of IAB categorised according to hypertension and BMI category were shown in figure 1 . The prevalence of IAB in hypertensive subjects was found to be higher than the normotensive in both men ( 9.5 vs $5.9 \% ; \mathrm{p}<0.001)$ and women (6.6 vs $3.6 \% ; \mathrm{p}<0.001)$. In addition, the prevalence of IAB demonstrated a sharp rise with advancing BMI in both men $(4.9 \%, 9.0 \%$ and $13.0 \%$ in those with $\mathrm{BMI}<24.0,24.0-27.9$ and $\geq 28.0 \mathrm{~kg} / \mathrm{m}^{2}$, respectively; $\mathrm{p}$ for trend $<0.001)$ and women $(3.5 \%, 5.9 \%$ and $6.9 \%$ in those with $\mathrm{BMI}<24.0,24.0-27.9$ and $\geq 28.0 \mathrm{~kg} / \mathrm{m}^{2}$, respectively; $\mathrm{p}$ for trend $<0.001)$. The prevalence of IAB for BMI category by hypertension was calculated and presented in figure 2. Our results demonstrated that the prevalence of IAB rose significantly with advancing BMI in both normotensive and hypertensive subjects ( $p$ for trend $<0.001)$. Furthermore, higher prevalence of IAB was detected in the hypertensive subgroup at each BMI category (all ps $<0.05$ ).

\section{Linear relationship of $\mathrm{BP}, \mathrm{BMI}$ with $\mathrm{P}$ wave duration}

Multivariate linear regression analyses were performed to elucidate the association of BP, BMI and $\mathrm{P}$ wave duration, which are presented in table 2. To better understand the complex effects of the numerous clinical factors associated with $\mathrm{P}$ wave duration, 4 sets of multivariate models were employed accordingly. In model 1 , factors such as BP, BMI, age, gender and race were included, with results demonstrating that SBP, DBP and BMI were all independently associated with $P$ wave duration $(\beta=0.03,0.11$ and 0.44 , respectively; all ps $<0.001)$. In model 2, additional variables such as FBG, plasma lipids, SUA, smoking, drinking, education, income, use of anti-arrhythmic medication, use of anti-hypertensive medication, mitral stenosis/regurgitation and history of MI and HF were adjusted accordingly, the results of which revealed that the independent associations were presented with a relatively lower $\beta$ (all ps $<0.001)$. In 
model 3, after LAD had been further adjusted, the linear regression coefficients were found to decline to $0.02,0.09$ and 0.25 for SBP, DBP and BMI, respectively (all ps <0.001). Finally, in model 4, while LVMI was added to the multivariate linear regression, SBP, DBP and BMI were still all found to be independently associated with $\mathrm{P}$ wave duration (all ps $<0.005$ ).

\section{Associations between hypertension, overweight/obesity and IAB}

In an attempt to further evaluate the associations of hypertension and overweight/obesity with IAB, we performed a series of multivariate logistic regression analyses, the results of which were shown in table 3 . In model 1 , factors such as hypertension, BMI categories, age, gender and race were included, with results demonstrating that hypertensive subjects exhibited higher odds of IAB than the normotensive subjects ( $\mathrm{OR}=1.42 ; 95 \% \mathrm{CI} 1.19$ to $1.68)$. Compared with individuals with a BMI $<24.0 \mathrm{~kg} /$ $\mathrm{m}^{2}$, higher odds of IAB were found in both overweight $(\mathrm{OR}=1.76 ; 95 \% \mathrm{CI} 1.46$ to 2.11$)$ and obese individuals ( $\mathrm{OR}=2.32 ; 95 \%$ CI 1.88 to 2.87). In model 2, additional variables of diabetes, plasma lipids, SUA, smoking, drinking, education, income, use of anti-arrhythmic medication, use of anti-hypertensive medication, mitral stenosis/regurgitation, history of MI and history of HF were adjusted accordingly, with the subsequent results obtained indicating that the independent associations preserved with relatively lower ORs (all ps <0.05). In model 3, after LAD had been further adjusted, the ORs decreased to $1.29,1.40$ and 1.64 for hypertension, overweight and obesity, respectively (all ps $<0.05$ ). Finally, LVMI was added in model 4 , and the ORs changed to $1.27,1.42$ and 1.67 (all ps $<0.05$ ).

\section{DISCUSSION}

The current study aimed to conduct the largest evaluation $(n=11264)$ of the potential factors associated with IAB in a general Chinese population. Our key findings indicated that IAB is significantly less prevalent in China compared with the American population. Our current study focused on obesity and hypertension, which are pandemic clinical issues that often coexist in people across the world. Our results identified a higher prevalence of IAB in subjects presenting with hypertension and advancing BMIs. Hypertension was further demonstrated to increase the prevalence of IAB among overweight and obesity individuals. Furthermore, we uncovered that both obesity and hypertension were significantly and independently associated with IAB, even after adjusting for multiple clinical covariates, echocardiographic LAD and LVMI.

An extremely low prevalence of IAB was detected during the current study in comparison to previous data, ${ }^{24}$ which may be attributed to the fact that our study population comprised of rural residents with a relatively young mean age. In addition, it is also noteworthy that the prevalence of AF in Asian population is considerably lower compared with Western populations. ${ }^{22}$ Based on the aforementioned analysis, we asserted that IAB might contribute to the relatively low prevalence of $\mathrm{AF}$ in China.

Some previous studies have reported the correlations of IAB with obesity ${ }^{23} 24$ and hypertension. ${ }^{25-28}$ Additionally, our results on the potential risk factors contributing to IAB are consistent with existing literatures reported in the ARIC ${ }^{16}$ and Multiethnic Study of Atherosclerosis (MESA) studies. ${ }^{29}$ The ARIC research demonstrated that IAB is significantly associated with obesity and hypertension in a cross-sectional population-based analysis, which are independent of age and cardiovascular risk factors. ${ }^{16}$ This was consistent with our findings of the current study. The subgroup analysis of the MESA study further confirmed our discoveries, illustrating the association of increased BMI with IAB after adjusting for age, sex, ethnicity and pericardial fat. ${ }^{29}$ However, the examination in both of these aforementioned two studies failed to adjust for factors such as LAD and LVMI. In contrast, a considerable strength of our current study was our comprehensive adjustment for echocardiographic LAD and LVMI, in addition to cardiovascular risk factors in the multivariable analyses.

Our key findings revealed that the associations of hypertension and obesity with IAB were independent of echocardiographic LAD and LVMI, indicating that a prolonged $\mathrm{P}$ wave may occur prior to left atrial enlargement or at least was not consistent. The aforementioned finding was supported by Antoni Bayés de Luna's study, who proposed the concept of 'Bayes' syndrome'. He stated that the potential pathophysiology of IAB was directly related to a block in the area of the Bachmann's bundle. Although atrial enlargement and IAB share a similar electrocardiographic pattern, they are two separate entities. ${ }^{13031}$ Another study highlighted that the mechanism underlying the associations of hypertension and obesity with IAB is likely to be multifactorial in nature. ${ }^{32}$ Obesity and hypertension have been demonstrated to increase cardiac preload, resulting in compensatory remodelling, ${ }^{33}$ with various reports indicating that obesity induces the expression of paracrine hormone with endovascular effects that may also alter atrial pressures and preload conditions. ${ }^{34}{ }^{35}$ The abovementioned findings elucidated the role of $\mathrm{LAD}$ in relation to the impact of obesity and hypertension on IAB. On the other hand, insulin resistance, a basic feature of metabolic syndrome, exhibits cellular and electrophysiologic effects by modifying metabolic function, including impairment of mitochondrial function and oxidative stress. ${ }^{36}$ Furthermore, obesity has been reported to directly drive electrophysiologic remodelling by altering the myocardial matrix secondary to adipose-derived hormones. ${ }^{37} 38$ Therefore, it can be summarised that the association of hypertension and obesity with IAB is not entirely dependent on LAD and LVMI, which serves as an explanation for our findings regarding the independent influence of hypertension and obesity on IAB. 


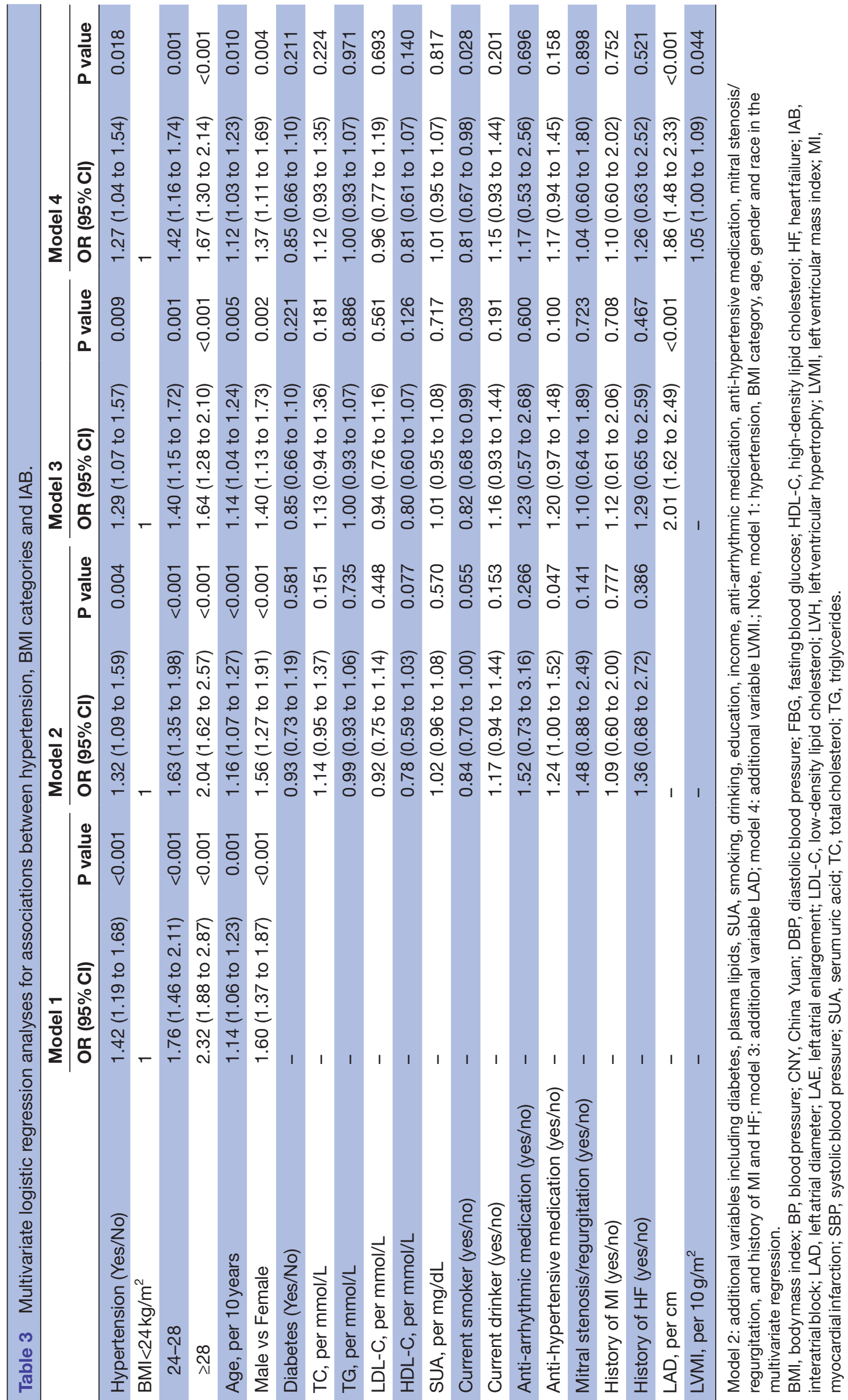


However, this study has several limitations. First, the cross-sectional design of our study was unable to examine the longitudinal associations and distinguish causality between hypertension, obesity and IAB. Second, the detailed information of ECG waveform recorded using automatic measures were unable to be read by the MUSE system, so we did not differentiate between partial and advanced IAB. Third, left atrial volume is a better index than LAD to estimate left atrial size according to the recommendations of the American Society of Echocardiography. ${ }^{21}$ However, as a large-scale epidemiological investigation, we only measured LAD in our current study. Therefore, it may not represent an accurate picture of left atrial size although this measurement has been used extensively in clinical practice and research. Fourth, the sample size in some subgroups by stage of hypertension in our study was relatively small. For example, the number of subjects with elevated BP stage and IAB was only 54, thus we did not perform subgroup analyses according to BP stages. Finally, all the enrolled participants were from the same province in China, resulting in limited representation.

\section{CONCLUSION}

The current study evidenced a relatively higher prevalence of IAB in subjects with hypertension and advancing BMIs. Hypertension additionally augmented the prevalence of IAB among individuals from each BMI category. Furthermore, it was uncovered that both hypertension and overweight/obesity significantly and independently increased the prevalence of IAB even after adjusting for multiple relevant covariates, echocardiographic LAD and LVMI.

Acknowledgements We would like to thank Professor Liqiang Zheng for his help with data collection and data management. We would also like to extend our gratitude to Professor Zhao Li for discussions and support on the project.

Contributors GZS collected the data, analysed and prepared the first draft of the manuscript. YZ supervised the data collection and reviewed the manuscript. NY coordinated the data collection. SJW did the data analyses. YXS conceived the study design, reviewed the final manuscript and serves as guarantor for the contents of this paper. All authors approved the final version.

Funding This study was funded by National Science and Technology Support Program of China (No. 2012BAJ18B08-7) and National Key Research and Development Program of China (No. 2017YFC1307600).

Competing interests None declared.

Patient consent for publication Not required.

Ethics approval The current study was performed under the approval of the Ethics Committee of China Medical University in Shenyang, China. All the procedures were conducted in strict accordance with its ethical standards.

Provenance and peer review Not commissioned; externally peer reviewed.

Data sharing statement Data are available upon reasonable request.

Open access This is an open access article distributed in accordance with the Creative Commons Attribution Non Commercial (CC BY-NC 4.0) license, which permits others to distribute, remix, adapt, build upon this work non-commercially, and license their derivative works on different terms, provided the original work is properly cited, appropriate credit is given, any changes made indicated, and the use is non-commercial. See: http://creativecommons.org/licenses/by-nc/4.0/.

\section{REFERENCES}

1. Bayés de Luna A, Platonov P, Cosio FG, et al. Interatrial blocks. A separate entity from left atrial enlargement: a consensus report. $J$ Electrocardiol 2012;45:445-51.

2. Asad N, Spodick DH. Prevalence of interatrial block in a general hospital population. Am J Cardiol 2003;91:609-10.

3. Chhabra L, Devadoss R, Chaubey VK, et al. Interatrial block in the modern era. Curr Cardiol Rev 2014;10:181-9.

4. Jairath UC, Spodick DH. Exceptional prevalence of interatrial block in a general hospital population. Clin Cardiol 2001;24:548-50.

5. Tse G, Wong CW, Gong M, et al. Predictive value of inter-atrial block for new onset or recurrent atrial fibrillation: A systematic review and meta-analysis. Int J Cardiol 2018;250:152-6.

6. Soliman EZ, Prineas RJ, Case LD, et al. Ethnic distribution of ECG predictors of atrial fibrillation and its impact on understanding the ethnic distribution of ischemic stroke in the Atherosclerosis Risk in Communities (ARIC) study. Stroke 2009;40:1204-11.

7. Magnani JW, Johnson VM, Sullivan LM, et al. P wave duration and risk of longitudinal atrial fibrillation in persons $\geq 60$ years old (from the Framingham Heart Study). Am J Cardiol 2011;107:917-21.

8. Goyal SB, Spodick DH. Electromechanical dysfunction of the left atrium associated with interatrial block. Am Heart $J$ 2001;142:823-7.

9. Lorbar M, Levrault R, Phadke JG, et al. Interatrial block as a predictor of embolic stroke. Am J Cardiol 2005;95:667-8.

10. Ariyarajah V, Apiyasawat $\mathrm{S}$, Najjar $\mathrm{H}$, et al. Frequency of interatrial block in patients with sinus rhythm hospitalized for stroke and comparison to those without interatrial block. Am J Cardiol 2007;99:49-52.

11. Ariyarajah $\mathrm{V}$, Puri $P$, Apiyasawat $S$, et al. Interatrial block: a novel risk factor for embolic stroke? Ann Noninvasive Electrocardiol 2007;12:15-20.

12. Wu JT, Wang SL, Chu YJ, et al. CHADS and $\mathrm{CHA}_{2} \mathrm{DS}_{2}$-VASc Scores Predict the Risk of Ischemic Stroke Outcome in Patients with Interatrial Block without Atrial Fibrillation. J Atheroscler Thromb 2017;24:176-84.

13. Magnani JW, Gorodeski EZ, Johnson VM, et al. $\mathrm{P}$ wave duration is associated with cardiovascular and all-cause mortality outcomes: the National Health and Nutrition Examination Survey. Heart Rhythm 2011;8:93-100.

14. Ariyarajah V, Apiyasawat $S$, Moorthi $R$, et al. Potential clinical correlates and risk factors for interatrial block. Cardiology 2006;105:213-8.

15. Ariyarajah V, Kranis M, Apiyasawat S, et al. Potential factors that affect electrocardiographic progression of interatrial block. Ann Noninvasive Electrocardiol 2007;12:21-6.

16. Magnani JW, Lopez FL, Soliman EZ, et al. P wave indices, obesity, and the metabolic syndrome: the atherosclerosis risk in communities study. Obesity 2012;20:666-72.

17. O'Brien E, Petrie J, Littler W, et al. The British Hypertension Society protocol for the evaluation of automated and semi-automated blood pressure measuring devices with special reference to ambulatory systems. J Hypertens 1990;8:607-19.

18. Whelton PK, Carey RM, Aronow WS, et al. 2017 ACC/AHA/AAPA ABC/ACPM/AGS/APhA/ASH/ASPC/NMA/PCNA Guideline for the Prevention, Detection, Evaluation, and Management of High Blood Pressure in Adults: A Report of the American College of Cardiology/ American Heart Association Task Force on Clinical Practice Guidelines. J Am Coll Cardiol 2018;71:e127-e248.

19. Zhou BF. Effect of body mass index on all-cause mortality and incidence of cardiovascular diseases--report for meta-analysis of prospective studies open optimal cut-off points of body mass index in Chinese adults. Biomed Environ Sci 2002;15:245-52.

20. World Health Organization and International Diabetes Fedaration. Definition and diagnosis of diabetes mellitus and intermediate hyperglycemia: report of a WHO/IDF consultation. Geneva, Switzerland: WHO, 2006.

21. Lang RM, Badano LP, Mor-Avi V, et al. Recommendations for cardiac chamber quantification by echocardiography in adults: an update from the American Society of Echocardiography and the European Association of Cardiovascular Imaging. J Am Soc Echocardiogr 2015;28:1-39.

22. Sun GZ, Guo L, Wang XZ, et al. Prevalence of atrial fibrillation and its risk factors in rural China: a cross-sectional study. Int $J$ Cardiol 2015;182:13-17.

23. Baranchuk A, Parfrey B, Lim L, et al. Interatrial block in patients with obstructive sleep apnea. Cardiol J 2011;18:171-5.

24. Liu T, Fu Z, Korantzopoulos P, et al. Effect of obesity on p-wave parameters in a Chinese population. Ann Noninvasive Electrocardiol 2010;15:259-63.

25. Wu JT, Fan XW, Yang HT, et al. Association Between $\mathrm{CHADS}_{2}$ Score and the Development of Interatrial Block. Int Heart J 2018;59:1261-5. 
26. Bernal E, Bayés-Genís A, Ariza-Solé A, et al. Interatrial block, frailty and prognosis in elderly patients with myocardial infarction. $J$ Electrocardiol 2018;51:1-7.

27. Escobar-Robledo LA, Bayés-de-Luna A, Lupón J, et al. Advanced interatrial block predicts new-onset atrial fibrillation and ischemic stroke in patients with heart failure: The "Bayes' Syndrome-HF" study. Int J Cardiol 2018;271:174-80.

28. Martínez-Sellés M, Massó-van Roessel A, Álvarez-García J, et al. Interatrial block and atrial arrhythmias in centenarians: Prevalence, associations, and clinical implications. Heart Rhythm 2016;13:645-51.

29. Babcock MJ, Soliman EZ, Ding J, et al. Pericardial fat and atrial conduction abnormalities in the Multiethnic Study of Atherosclerosis (MESA). Obesity 2011;19:179-84.

30. Bacharova L, Wagner GS. The time for naming the Interatrial Block Syndrome: Bayes Syndrome. J Electrocardiol 2015;48:133-4.

31. Baranchuk A, Block I. Supraventricular Arrhythmias: Clinical Implications of Bayés' Syndrome. Minneapolis, MN: Cardiotext Publishing, 2017.

32. Sweeney G. Cardiovascular effects of leptin. Nat Rev Cardiol 2010;7:22-9.
33. Abel ED, Litwin SE, Sweeney G. Cardiac remodeling in obesity. Physiol Rev 2008;88:389-419.

34. Qasim A, Mehta NN, Tadesse MG, et al. Adipokines, insulin resistance, and coronary artery calcification. J Am Coll Cardiol 2008;52:231-6

35. Martin SS, Qasim A, Reilly MP. Leptin resistance: a possible interface of inflammation and metabolism in obesity-related cardiovascular disease. J Am Coll Cardiol 2008;52:1201-10.

36. Anderson EJ, Kypson AP, Rodriguez E, et al. Substrate-specific derangements in mitochondrial metabolism and redox balance in the atrium of the type 2 diabetic human heart. J Am Coll Cardiol 2009;54:1891-8.

37. You T, Nicklas BJ, Ding J, et al. The metabolic syndrome is associated with circulating adipokines in older adults across a wide range of adiposity. J Gerontol A Biol Sci Med Sci 2008;63:414-9.

38. Schram K, Sweeney G. Implications of myocardial matrix remodeling by adipokines in obesity-related heart failure. Trends Cardiovasc Med 2008;18:199-205. 